Draft Version June 15, 2017

Preprint typeset using $\mathrm{LAT}_{\mathrm{E}} \mathrm{X}$ style emulateapj v. 12/16/11

\title{
FIRST RESULTS FROM THE LYMAN ALPHA GALAXIES IN THE EPOCH OF REIONIZATION (LAGER) SURVEY: COSMOLOGICAL REIONIZATION AT $Z \sim 7$
}

\author{
Zhen-Ya Zheng ${ }^{1,2,3}$, Junxian Wang ${ }^{4}$, James Rhoads ${ }^{5,6}$, Leopoldo Infante $^{2}$, Sangeeta Malhotra ${ }^{5,6}$, Weida Hu ${ }^{4}$, \\ Alistair R. Walker ${ }^{7}$, Linhua Jiang ${ }^{8}$, Chunyan Jiang $^{1,3,9}$, Pascale Hibon $^{10}$, Alicia Gonzalez $^{5}$, Xu Kong ${ }^{4}$, \\ XianZhong Zheng ${ }^{11}$, Gaspar Galaz ${ }^{2}$, L. Felipe Barrientos ${ }^{2}$. \\ ${ }^{1}$ CAS Key Laboratory for Research in Galaxies and Cosmology, Shanghai Astronomical Observatory, Shanghai 200030, China; \\ zhengzy@shao.ac.cn \\ ${ }^{2}$ Institute of Astrophysics and Center for Astroengineering, Pontificia Universidad Catolica de Chile, Santiago 7820436, Chile; \\ linfante@astro.puc.cl \\ ${ }^{3}$ Chinese Academy of Sciences South America Center for Astronomy, Santiago 7591245, Chile \\ ${ }^{4}$ CAS Key Laboratory for Research in Galaxies and Cosmology, Department of Astronomy, University of Science and Technology of \\ China, Hefei, Anhui 230026, China; jxw@ustc.edu.cn \\ ${ }^{5}$ School of Earth and Space Exploration, Arizona State University, Tempe, AZ 85287, USA; Sangeeta.Malhotra@asu.edu, \\ James.Rhoads@asu.edu \\ ${ }^{6}$ Astrophysics Science Division, Goddard Space Flight Center, 8800 Greenbelt Road, Greenbelt, Maryland 20771, USA; \\ ${ }^{7}$ Cerro Tololo Inter-American Observatory, Casilla 603, La Serena, Chile \\ ${ }^{8}$ The Kavli Institute for Astronomy and Astrophysics, Peking University, Beijing, 100871, China \\ ${ }^{9}$ Núcleo de Astronomía, Facultad de Ingeniería y Ciencias, Universidad Diego Portales, Av. Ejército 441, Santiago, Chile \\ ${ }^{10}$ European Southern Observatory, Alonso de Cordova 3107, Casilla 19001, Santiago, Chile \\ ${ }^{11}$ Purple Mountain Observatory, Chinese Academy of Sciences, Nanjing 210008, China
}

Draft version June 15, 2017

\begin{abstract}
We present the first results from the ongoing LAGER project (Lyman Alpha Galaxies in the Epoch of Reionization), which is the largest narrowband survey for $z \sim 7$ galaxies to date. Using a specially built narrowband filter NB964 for the superb large-area Dark-Energy Camera (DECam) on the NOAO/CTIO 4m Blanco telescope, LAGER has collected 34 hours NB964 narrowband imaging data in the $3 \mathrm{deg}^{2}$ COSMOS field. We have identified 23 Lyman Alpha Emitter (LAE) candidates at $z=$ 6.9 in the central 2- $\mathrm{deg}^{2}$ region, where DECam and public COSMOS multi-band images exist. The resulting luminosity function can be described as a Schechter function modified by a significant excess at the bright end ( 4 galaxies with $\mathrm{L}_{L y \alpha} \sim 10^{43.4 \pm 0.2} \mathrm{erg} \mathrm{s}^{-1}$ ). The number density at $\mathrm{L}_{L y \alpha} \sim 10^{43.4 \pm 0.2}$ $\mathrm{erg} \mathrm{s}^{-1}$ is little changed from $z=6.6$, while at fainter $\mathrm{L}_{L y \alpha}$ it is substantially reduced. Overall, we see a fourfold reduction in Ly $\alpha$ luminosity density from $z=5.7$ to 6.9. Combined with a more modest evolution of the continuum UV luminosity density, this suggests a factor of $\sim 3$ suppression of Ly $\alpha$ by radiative transfer through the $z \sim 7$ intergalactic medium (IGM). It indicates an IGM neutral fraction $x_{H I} \sim 0.4-0.6$ (assuming Ly $\alpha$ velocity offsets of $100-200 \mathrm{~km} \mathrm{~s}^{-1}$ ). The changing shape of the Ly $\alpha$ luminosity function between $z \lesssim 6.6$ and $z=6.9$ supports the hypothesis of ionized bubbles in a patchy reionization at $z \sim 7$.
\end{abstract}

Keywords: cosmology: observations — dark ages, reionization, first stars — galaxies: high-redshift galaxies: luminosity function, mass function

\section{INTRODUCTION}

In the last decade, much progress has been made in narrowing down the epoch of cosmological reionization to be between $z \sim 6$ ( Ly $\alpha$ saturation in $\mathrm{z} \sim 6$ quasar, Fan et al. 2006) and $z \sim 9$ (Polarization of CMB photons, Planck results XIII 2015). In between, quasars, gamma ray bursts (GRBs), and galaxies at $z \gtrsim 6$ are important to constrain the nature of reionization. However, the rarity of high- $z$ quasars (with only one known at $z \gtrsim 7$, Bolton et al. 2011), and of high- $z$ GRBs with rapid followup, limit their application as reionization probes. In contrast, hundreds of galaxies at $z \gtrsim 6$ have been reported from ground-based narrowband Ly $\alpha$ line searches (e.g., Ouchi et al. 2010), from space-based broadband Lyman break searches (e.g., Bouwens et al. 2015; Finkelstein et al. 2015), and from newly taken space-based grism spectroscopic surveys (Tilvi et al. 2016; Bagley et al. 2017). Their luminosities, number densities, clustering, and ionizing powers are essential to probe the epoch of reionization $(E o R)$.

Because Ly $\alpha$ photons are resonantly scattered by neutral hydrogen, Ly $\alpha$ emitters (LAEs) provide a sensitive, practical, and powerful tool to determine the epoch, duration, and inhomogeneities of reionization. With a sample of LAEs in EoR, the easiest Ly $\alpha$ reionization test is the luminosity function (LF) test. Rhoads \& Malhotra (2001) first applied this test at $z=5.7$, then at 6.5 (Malhotra \& Rhoads 2004; Stern et al. 2005). Subsequent surveys have confirmed and refined the $z=6.5-6.6$ neutral fraction measurement to $x_{H I} \lesssim 0.3$ (Ouchi et al. 2010; Kashikawa et al. 2011), and have found a significant neutral fraction increase from $\mathrm{z}=6.6$ to $\mathrm{z}$ $=7.3$ (Konno et al. 2014). Other implementations of Ly $\alpha$ reionization tests include the $\operatorname{Ly} \alpha$ visibility test (Jensen et al. 2013; Schenker et al. 2014; Faisst et al. 2014), i.e., the Ly $\alpha$ fraction in LBGs in EoR, the volume test (Malhotra \& Rhoads 2006, Rhoads \& Malhotra 2017 , in prep.) , i.e., each Ly $\alpha$ galaxy is taken as evi- 
dence for a certain volume of ionized gas, and the clustering test, e.g., the signature of a patchy partially-ionized IGM is sought by looking for excess spatial correlations in the Ly $\alpha$ galaxy distribution (Furlanetto et al. 2006; McQuinn et al. 2007; Jensen et al. 2013).

Redshift $z \gtrsim 7$ is the frontier in Ly $\alpha$ and re-ionization studies. Many searches for LAEs at $\mathrm{z}=6.9-7.0$ (Iye et al. 2006; Ota et al. 2008, 2010; Hibon et al. 2011, 2012), $\mathrm{z}=7.2-7.3$ (Shibuya et al. 2012; Konno et al. 2014), and $\mathrm{z}=7.7$ (Hibon et al. 2010; Tilvi et al. 2010; Krug et al. 2012) have yielded two dozen narrowband-selected candidates, but only 3 of these have been spectroscopically confirmed so far (Iye et al. 2006; Rhoads et al. 2012; Shibuya et al. 2012). In comparison, hundreds of LAEs have been found at lower redshift, at $z=6.6$ (e.g., Hu et al. 2010; Ouchi et al. 2010; Kashikawa et al. 2011; Matthee et al. 2015), and a large fraction of them have been spectroscopically confirmed (i.e., Kashikawa et al. 2011). The comoving volumes of the searches for $z \gtrsim 7$ LAEs are all less than $6 \times 10^{5}$ $\mathrm{cMpc}^{3}$, which are more likely to be affected by cosmic variance. In fact cosmic variance is more important for the observability of LAEs in patchy reionization. Further more, some of the surveys with $200 \AA$ wide filters in the far-red inevitably (e.g., Ota et al. 2008, 2010) include one or two weak $\mathrm{OH}$ emission lines, limiting their sensitivity. Thus, there is an urgent need for a systematic survey at $\mathrm{z} \sim 7$ with sufficient volume and depth to determine if the Ly $\alpha$ line is attenuated due to neutral IGM in a statistically significant way.

In this Letter, we report the discovery of a population of candidate $z \sim 7 \operatorname{Ly} \alpha$ emitters in the first field (COSMOS) of the Lyman-Alpha Galaxies in the Epoch of Reionization (LAGER) survey. With LAGERCOSMOS, we have the largest sample to date of candidate $z \sim 7$ LAEs. In $\S 2$, we describe the observation and data reduction of LAGER, then introduce the candidates selection method. In $\S 3$, we present the candidates and their Ly $\alpha$ LF at $z \sim 7$. In $\S 4$, we discuss the implications of these discoveries for $E o R$ at $z \sim 7$.

\section{SURVEY DESCRIPTION AND DATA}

\subsection{LAGER Survey}

The LAGER survey is the largest narrowband survey yet for LAEs at $z \gtrsim 7$. It is currently ongoing, using the Dark Energy Camera (DECam, with FOV $\sim 3 \mathrm{deg}^{2}$ ) on the NOAO-CTIO $4 \mathrm{~m}$ Blanco telescope together with an optimally designed custom narrowband filter NB964 ${ }^{1}$ (Central wavelength $\sim 9642 \AA$, FWHM $\sim 90 \AA$ ). LAGER is designed to select more than a hundred $\mathrm{z} \sim 7 \mathrm{LAEs}$ over an area of $12 \mathrm{deg}^{2}$ in 4 fields $\left(8 \times 10^{6} \mathrm{cMpc}^{3}\right)$. Currently, LAGER has collected 47 hrs NB964 narrowband imaging in 3 fields (CDF-S, COSMOS, and DLS-f5) taken during 9 nights in 2015 December, 2016 February, March and November.

The deepest NB964 imaging is done in COSMOS, where we have obtained 34 hours' NB964 exposure in a $3 \mathrm{deg}^{2}$ field. The exposure time per NB964 frame is 900 s. Consecutive exposures were dithered by $\sim 100^{\prime \prime}$ so that chip gaps and bad pixels do not lead to blank areas

1 Please see the filter information at NOAO website: http://www.ctio.noao.edu/noao/content/Properties-N964-filter in the final stacks. We also took $\sim 0.5-1$ hrs $\mathrm{z}$ and $\mathrm{Y}$ bands exposure per field to exclude possible transients. There are deep archival DECam and Subaru broadband images in COSMOS.

\subsection{Data Reduction and Analysis}

We downloaded the reduced and calibrated DECam resampled images from the NOAO Science Archive, which were processed through the NOAO Community Pipeline (version 3.7.0, Valdes et al. 2014). The individual frames were stacked following the weighting method in Annis et al. (2014) with SWarp (version 2.38.0, Bertin 2010). The seeing of the final stacked LAGER-COSMOS narrowband image is $0.93^{\prime \prime}$.

The zero-magnitudes of the DECam images were calibrated to the Subaru Suprime-Cam broadband magnitudes of the stars in the public COSMOS/UltraVISTA $K_{S}$-selected catalog (Muzzin et al. 2013). We estimated the image depth by measuring the root mean square (rms) of the background in blank places where detected $(>3 \sigma)$ signals were masked out. In a $2 "$ diameter aperture, the $3 \sigma$ limiting $\mathrm{AB}$ magnitudes of the DECam images are $[u, g, r, i, z, Y, \mathrm{NB} 64]_{3 \sigma}=[26.2,27.4$, 26.9, 26.6, 26.1, 24.3, 25.6]. Deep Subaru Suprime-Cam broad and narrow band images are available in the central 2- $\operatorname{deg}^{2}$ area. We downloaded the raw images from the archival server SMOKA (Baba et al. 2002), and produced our own broad and narrow band stacked images (follow-up work of Jiang et al. 2013). Their $3 \sigma$ limiting magnitudes within a $2^{\prime \prime}$ diameter aperture are $\left[B, g^{\prime}, V\right.$, $\left.r^{\prime}, i^{\prime}, z^{\prime}, \mathrm{NB} 711, \mathrm{NB} 816, \mathrm{NB} 921\right]_{3 \sigma} \sim[27.9,27.6,27.2$, 27.4, 27.3, 25.9, 26.0, 26.1, 26.2]. These Subaru SuprimeCam images are deeper and have better seeing than the DECam images on average.

We calculated the narrowband completeness via Monte Carlo simulations with the BALROG software (Suchyta et al. 2016). The completeness fraction is defined as the SExtractor recovery percentage of the randomly distributed artificial sources in NB964 image as a function of narrowband magnitude. For point sources, the narrowband NB964 aperture magnitudes corresponding to the $80 \%, 50 \%$ and $30 \%$ completeness fractions are 24.2, 25.0, and 25.3, respectively. For pseudo LAEs, we choose fake sources similar to that used in Konno et al. (2017), which have a Sérsic profile with the Sérsic index of $n=1.5$, and the half-light radius of $r_{e} \sim 0.9 \mathrm{kpc}$ $(0.17$ arcsec at $z=6.9)$. The narrowband NB964 aperture magnitudes corresponding to the $80 \%, 50 \%$ and $30 \%$ completeness fractions are $24.3,24.7$, and 25.0 for pesudo LAEs, respectively.

\subsection{Selection Criteria for $z \sim 7 L A E s$}

Since Subaru broadband images are deeper and have better seeings than the DECam broadband images, we only apply selection criteria for $\mathrm{z} \sim 7 \mathrm{LAEs}$ in the central 2-deg ${ }^{2}$ area covered by both Suprime-Cam and DECam. The selection criteria for $z \sim 7$ LAEs include narrowband NB964 with $\geq 5-\sigma$ detection (NB964 $\left.\leq \mathrm{NB}_{56}=25.07\right)$, narrowband NB964 excess over broadband z $(\mathrm{z}-\mathrm{NB} 964 \geq 1.0)^{2}$, and non-detections in both DECam ugri and Suprime-Cam $B g^{\prime} V r^{\prime} i^{\prime}$, N711,

2 As NB964 lies to the edge of $\mathrm{z}$ band transmission curve, very red continuum sources may mimic emission line galaxies. We ex- 

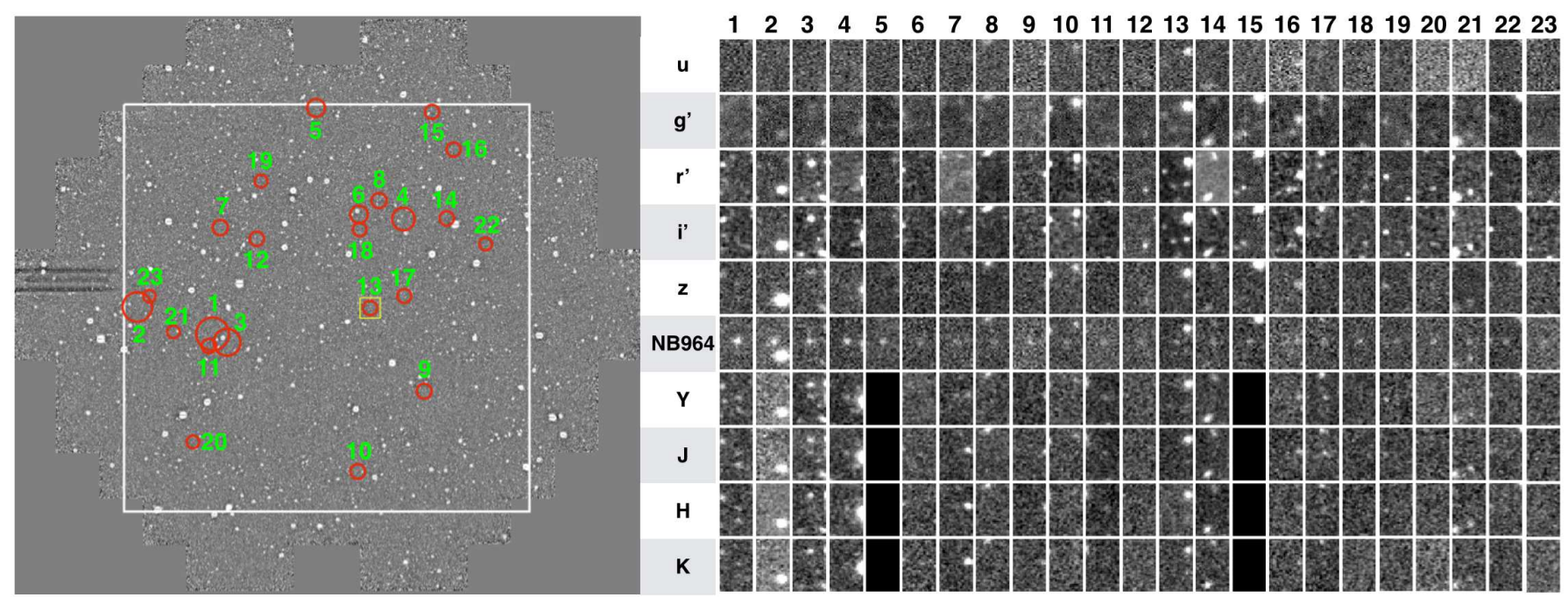

Figure 1. Left: The DECam NB964 COSMOS field showing uneven distribution of candidate $z \sim 7$ LAEs. The DECam NB964 area is $\sim 3 \mathrm{deg}^{2}$, and the overlap region with Subaru Suprime-Cam multi-band images is the central 2 deg$^{2}$ area in the white box. We have selected 23 candidate $z \sim 7$ LAEs, which are marked in red circles with aperture sizes scaled to their Ly $\alpha$ line fluxes and normalized to $\sim$ $1 \mathrm{pMpc}$ for the brightest LAE. The only spectroscopically confirmed LAE from previous narrowband survey (Rhoads et al. 2012) in this field is recovered and marked by a yellow box. Right: Optical and NIR thumbnail images of the 23 candidate LAEs. We select to display images of DECam $u$, Suprime-Cam $g^{\prime} r^{\prime} i^{\prime}$, DECam $z$, DECam NB964, and UltraVISTA (McCracken et al. 2012, DR3) YJHK $K_{S}$ bands. The stamp size is $10^{\prime \prime} \times 5^{\prime \prime}$.

N816, N921 bands. We specially exclude broadband detections in the $1^{\prime \prime} .08$ (4 pixel) diameter aperture to exclude marginal signals in these bands. 156 sources passed the selection criteria, but a large fraction of them are fake sources (bleed trails, diffraction spikes, etc) after our visual check on NB964 image. With our team's tripartite visual check on both NB964 and broadband images, 27 candidate $\mathrm{z} \sim 7$ galaxies are selected. No transient was identified among them using available broadband images.

\section{RESULTS}

\subsection{Candidate LAEs at $z \sim 7$ in LAGER-COSMOS}

We estimate the Ly $\alpha$ line fluxes and EWs of the candidates using NB and broadband photometry ${ }^{3}$. We further exclude 4 out of the 27 candidates with estimated line $\mathrm{EW}<10 \AA$.

In total, 23 candidate $\mathrm{z} \sim 7 \mathrm{LAEs}$, which have Ly $\alpha$ line fluxes in the range of $0.8-7.8 \times 10^{-17} \mathrm{erg} \mathrm{cm}^{-2} \mathrm{~s}^{-1}$ and rest-frame equivalent widths $\mathrm{EW}_{R} \geq 10 \AA$, are selected. The corresponding observed Ly $\alpha$ line luminosities are $5.4-43.4 \times 10^{42} \mathrm{erg} \mathrm{s}^{-1}$. Their positions and their multi-band thumbnail images are plotted in Figure 1.

The 4 brightest of them, with $\mathrm{L}(\mathrm{Ly} \alpha)=19.3-43.4$ $\times 10^{42} \mathrm{ergs}^{-1}$, are the most luminous candidate LAEs known at $z \gtrsim 7$, thanks to the large survey volume of LAGER. We have confirmed 3 of the brightest LAEs at $z \sim 7$ (Hu et al. 2017) with our recent Magellan/IMACS spectroscopic follow-up observations.

amine the $\mathrm{z}-\mathrm{NB}$ vs $\mathrm{z}-\mathrm{Y}$ plot for bright NB detected sources, and find red sources with $\mathrm{z}-\mathrm{Y} \sim 1.0 \mathrm{mag}$ have $\mathrm{z}-\mathrm{NB} \sim 0.4 \mathrm{mag}$, thus such effect is not important.

${ }^{3}$ UltraVISTA Y band (McCracken et al. 2012, DR3) is used to directly estimate the UV continuum of LAGER LAEs. For those without UltraVISTA Y band coverage, we use DECam z band and follow the Appendix of Zheng et al. (2016) to estimate the Ly $\alpha$ line fluxes and EWs. For broadband non-detections, $2 \sigma$ upper limits are chosen. We assume a flat UV continuum of $f_{v} \propto v^{0}$ and the IGM absorption from Madau (1995) in the above calculations. For candidates with $z$ or $Y$ band detection, such assumption holds within statistical uncertainty.
One of the candidates, J09:59:50.99+02:12:19.1, was previously selected and spectroscopically confirmed as a $z=6.944$ LAE with Magellan IMACS narrowband imaging and spectroscopy over a much smaller field (Rhoads et al. 2012). It is well recovered with our selection using new DECam narrowband imaging.

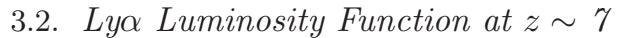

We use the $V / V_{\max }$ method to calculate the Ly $\alpha$ LF at $z=6.9$. The formula below is adopted:

$$
\Phi(L) d L=\sum_{L_{i} \in[L-\Delta L / 2, L+\Delta L / 2]} \frac{1}{V_{\max } f_{\text {comp }}\left(N B_{i}\right)} .
$$

Here $\mathrm{V}_{\max }$ is simply a constant, the maximum survey volume for LAEs $\left(\mathrm{V}_{\max }=1.26 \times 10^{6} \mathrm{cMpc}^{3}\right.$ calculated from sky coverage and NB964 central wavelength/FWHM), and $f_{\text {comp }}\left(N B_{i}\right)$ is the detection completeness for sources with narrowband magnitude $\mathrm{m}\left(\mathrm{NB}_{i}\right)$ interpolated in the narrowband detection completeness for fake LAEs (see §2.2).

Table 1

Ly $\alpha$ luminosity function at $z \sim 7$ from LAGER-COSMOS field.

\begin{tabular}{cccc}
\hline \hline $\log _{10}\left(\mathrm{~L}_{i}\right)$ & $\mathrm{N} \pm \Delta \mathrm{N}^{\ddagger}$ & $\left\langle f_{\text {comp. }}\right\rangle$ & $\begin{array}{c}\log _{10} \Phi\left(L_{i}\right) \\
(\Delta L=0.125)\end{array}$ \\
\hline $\left.\mathrm{L}_{i} \pm \frac{\Delta L}{2}\right]$ & & {$\left[\left(d \log _{10} L\right)^{-1} \mathrm{Mpc}^{-3}\right]$} \\
\hline 42.71 & $6_{-2.4}^{+3.6}$ & 0.21 & $-3.74_{-0.22}^{+0.20}$ \\
42.84 & $7_{-2.6}^{+3.8}$ & 0.44 & $-4.00_{-0.20}^{+0.19}$ \\
42.96 & $4_{-1.9}^{+3.2}$ & 0.56 & $-4.34_{-0.28}^{+0.25}$ \\
43.09 & $2_{-1.3}^{+2.7}$ & 0.80 & $-4.80_{-0.45}^{+0.37}$ \\
43.21 & $<2.6$ & 0.82 & $<-5.10$ \\
43.33 & $1_{-0.8}^{+2.3}$ & 0.85 & $-5.13_{-0.77}^{+0.52}$ \\
43.46 & $2_{-1.3}^{+2.7}$ & 0.88 & $-4.84_{-0.45}^{+0.37}$ \\
43.59 & $1_{-0.8}^{+2.3}$ & 0.90 & $-5.15_{-0.77}^{+0.52}$ \\
\hline.
\end{tabular}

The Ly $\alpha$ LF of LAEs at $z \sim 7$ in the LAGERCOSMOS field is listed in Table 1 , and plotted in Figure 

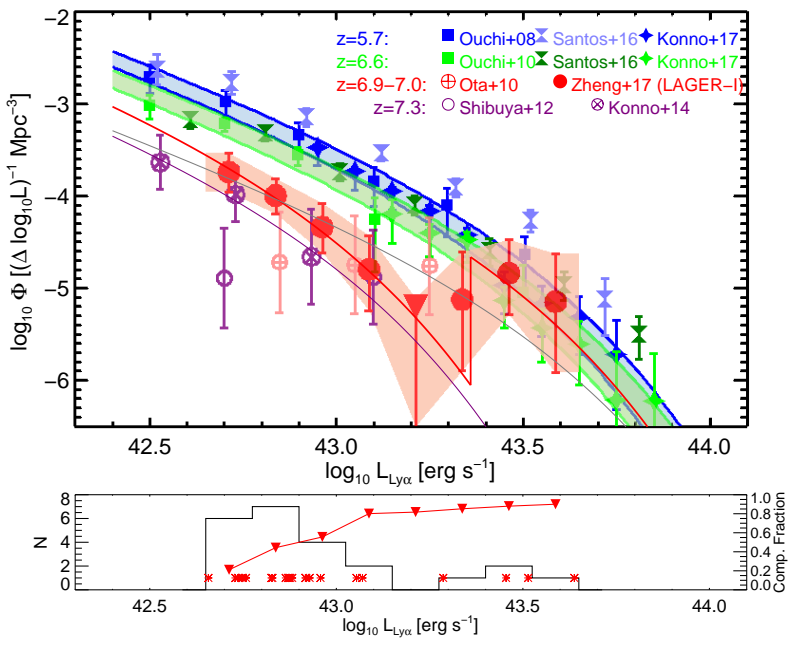

Figure 2. Top: The Ly $\alpha$ LFs of LAE surveys at $z \sim[5.7,6.6$, 7, 7.3]. The red filled circles show our results at $z \sim 7$ from LAGER-COSMOS field. References: Ly $\alpha$ LFs at $\mathrm{z}=5.7$ from Ouchi et al. (2008) (blue filled squares), Konno et al. (2017) (blue filled stars), and Santos et al. (2016) (light-blue filled hourglass), at $\mathrm{z}=6.6$ from Ouchi et al. (2010) (green filled squares), Konno et al. (2017) (green filled stars), and Santos et al. (2016) (dark-green filled hourglass), at $z \sim 7$ from this work (red filled circles) and Ota et al. (2010) (pink empty circles with plus), and at $\mathrm{z}=7.3$ from Shibuya et al. (2012) (purple empty circles) and Konno et al. (2014) (purple empty circles with cross). The colored regions in blue and green show the best Schechter fit with $\pm 1 \sigma$ error in $L *$ at $z \sim 5.7$ and 6.6, respectively, from Konno et al. (2017). The solid and dashed lines in red (gray) show the best Schechter fits at $z \sim 6.9$ with $L$ range $42.65 \leq \log _{10} L<43.25\left(42.65 \leq \log _{10} L \leq\right.$ 43.65) (see Table 2). A truncated Schechter function is also plotted (red solid line, see text for details) to measure the bright end LF bump. The purple line shows the best Schechter fit at $z \sim 7.3$ from Konno et al. (2014). All these Schechter fits have a fixed faint-end slope $\alpha=-2.5$. Bottom: The histogram of Ly $\alpha$ luminosities of LAGER LAEs. The average completeness fraction for our LAEs interpolated from Section 2.2 is also plotted as a red solid line with red triangles.

2 together with LAE LFs at $z \sim 5.7-7.3$ from literature. The LF shows dramatic evolution not only in normalization, but also in the shape.

The relatively fainter end of our LF $\left(42.65 \leq \log _{10} L_{\text {Ly }}\right.$ $\leq 43.25$ ) at $z \sim 7$ can be fitted with a a single Schechter function in the form of

$$
\Phi(L) d L=\Phi^{*}\left(\frac{L}{L^{*}}\right)^{\alpha} \exp \left(-\frac{L}{L^{*}}\right) d\left(\frac{L}{L^{*}}\right) .
$$

Because our survey does not go far below $L^{*}$, we fix $\alpha=-2.5$ in our fitting, which is suggested from recent studies on Ly $\alpha$ LFs at $z=5.7$ and $z=6.6$ by Konno et al. (2017). Konno et al. (2017) report the largest narrowband surveys to date for LAEs at $z=5.7$ and $z=6.6$ taken with Subaru HSC on the $14-21 \mathrm{deg}^{2}$ sky, and their best-fit Schechter function analyses also include the smaller but much deeper narrowband surveys taken with Subaru Suprime-Cam (Ouchi et al. 2008, 2010) at the corresponding redshifts. Since the Ly $\alpha$ LFs by Konno et al. (2017) are derived from the largest LAE samples at $z=5.7$ and $z=6.6$ with the widest luminosity range, and we have consistent selection and analysis methods, we choose their Ly $\alpha$ LFs for comparison in the following sections. At $z>7$, we choose the Ly $\alpha$ LF at $z=7.3$ by Konno et al. (2014) for comparison. The bestfit Schechter function parameters of these LFs are listed
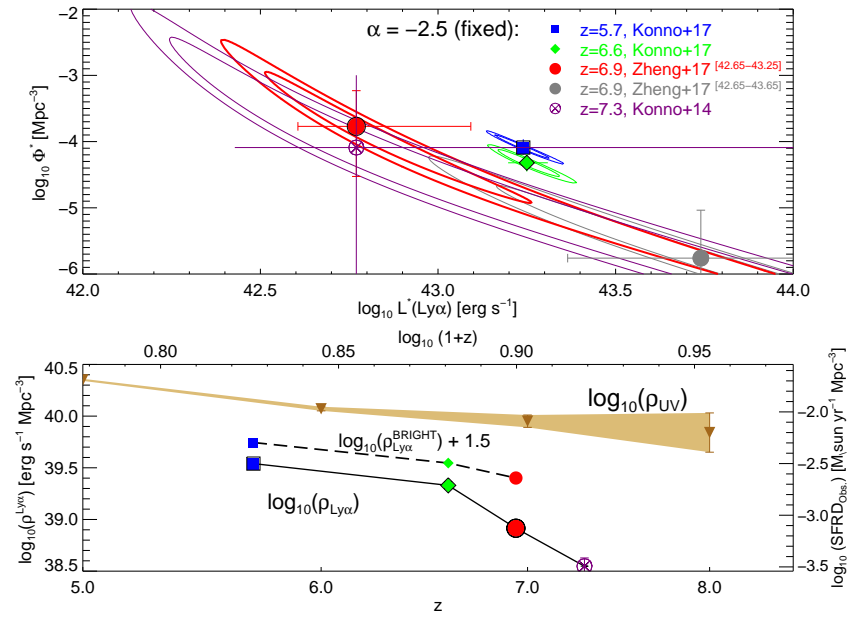

Figure 3. Top: The evolution of best-fit schechter parameters $L_{L y \alpha}^{*}$ and $\Phi_{L y \alpha}^{*}$ of Ly $\alpha$ LFs at $z=[5.7,6.6,6.9,7.3]$ with 1 and $2 \sigma$ error contours. Bottom: Evolution of Ly $\alpha$ and UV luminosity densities at $z \geq 6$. We plot the Ly $\alpha$ luminosity densities integrated over the best-fit Schechter functions $\left(42.4<\log _{10} L_{L y \alpha}<44.0\right)$, and at the bright-end $\left(43.3<\log _{10} L_{L y \alpha}<43.7\right)$. Please see Table 2 for the values. Note the bright luminosity density is scaled up by 1.5 dex. The yellow shaded region represents the UV luminosity densities for $L_{U V}>0.03 L_{U V}^{*}$ given by Finkelstein et al. (2015) at $z \sim 5.0,6.0,7.0$ and 8.0. The symbols are the same as those in Figure 2.

in Table 2, and plotted in the upper panel of Figure 3. We find a large drop in $\Phi$ of $\mathrm{Ly} \alpha \mathrm{LF}$, compared with that at $z=5.7$ and $z=6.6$. In the relatively fainter end, our Ly $\alpha$ LF at $z \sim 7$ is in agreement within the $1 \sigma$ measurement uncertainties of that at $z=7.3$.

More strikingly, we see a clear bump, i.e., significant excess to the Schechter function in the bright end of our $z \sim 7 \mathrm{LF}\left(\log _{10} L_{L y \alpha} \gtrsim 43.25\right)$. It demonstrates that, while the space density of faint LAEs drops tremendously from $z=5.7$ and 6.6 to $z=6.9$, that of the luminous ones shows no significantly change. Similarly but much less prominently, the LF evolution between $z=$ 5.7 and $z=6.6$ may also be differential, with a significant decline at the faint end but not clear evolution at the bright end (Matthee et al. 2015; Santos et al. 2016; Konno et al. 2017). In Fig. 2 we also plot a scaled-down and truncated version (red solid line at high luminosity end) of Ly $\alpha$ LF at $z=5.7$ from Konno et al. (2017), normalized by the UV luminosity density evolution between $z=5.7$ and $z=6.9^{4}$, which appears well consistent with our LF at the bright end within statistical uncertainties.

\section{DISCUSSION}

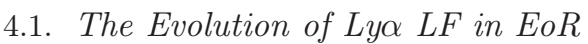

Cosmological reionization was well under way by $z \sim$ 9 (Planck results XIII 2015), and ended by $z \sim 6$ (Fan et al. 2006). Ly $\alpha$ galaxies at $z \gtrsim 6$, e.g., from LAE surveys at $z=5.7$ (Ouchi et al. 2008; Santos et al. 2016; Konno et al. 2017), at $z=6.6$ (Ouchi et al. 2010; Matthee et al. 2015; Konno et al. 2017), at $z=6.9$ (our LAGER-COSMOS sample in this work and Ota et al. 2017), and at $z=7.3$ (Konno et al. 2014), are unique samples useful to probe both galaxy evolution and reion-

4 Using $\rho_{z=6.9}^{U V} / \rho_{z=5.7}^{U V}$ interpolated from Finkelstein et al. (2015). 
ization.

In this work, we detect for the first time a significant bump at the bright end of $\operatorname{Ly} \alpha \operatorname{LF}$ at $z \gtrsim 7$. Previous surveys for LAEs at $z \gtrsim 7$ failed to reveal it as they covered much smaller volumes.

The existence of such bright end bump is consistent with the scenario proposed by Haiman \& Cen (2005), that bright LAEs are less attenuated by a neutral IGM than faint LAEs, as the larger Strömgren sphere surrounding luminous LAEs alleviates the neutral IGM absorption (also see Santos et al. 2016; Konno et al. 2017). Therefore, the evolution in the bright end of the LF better reflects the intrinsic evolution of LAEs, while the faint end is controlled by the evolution of both galaxies and the IGM.

The bottom panel of Figure 3 shows the evolution of luminosity densities both for Ly $\alpha$ and UV photons. Compared to LBGs (yellow shaded region), an accelerated evolution of LAE $L y \alpha$ densities from $\mathrm{z}=6.6$ (green diamonds) to $\mathrm{z}=7.3$ (purple cross), as reported by Konno et al. (2014), is clearly seen in Figure 3. Since UV photons detected in LBGs are insensitive to the neutral hydrogen in EoR, Konno et al. (2014) concluded that such rapid evolution in LAE LFs can be attributed to a large neutral IGM fraction at $z \sim 7.3$. Our LAGER survey shows rapid evolution from $z=6.6$ to 6.9 , and a smaller difference between $z=6.9$ and 7.3 (see Figure $3)$. It is possible that the evolution in the neutral fraction accelerated after $z=6.9$, though a smooth, monotonic neutral fraction evolution at $6.6 \leq z \leq 7.3$ also fits the LAGER results and Konno et al. (2014) measurements within their uncertainties. Note that the cosmic time from $z \sim 6.6$ to 6.9 and from $z \sim 6.9$ to 7.3 are approximately equal, which is about 50 Myr.

Furthermore, compared to the evolution of Ly $\alpha$ densities $\rho_{L y \alpha}$ between redshifts of 5.7 and 6.9, the evolution in the bright end only $\rho_{\text {Ly } \alpha}^{\text {Bright }}$ follows a much smoother trend, somehow similar to that of the UV densities of LBGs (bottom panel of Figure 3). This demonstrates that either the intrinsic LAE LF evolves moderately between $\mathrm{z}=5.7$ and 6.9 , or IGM attenuation plays a role even at the highest luminosity bin. Consequently, the dramatic and rapid evolution between $z=6.6$ and 6.9 in the faint end can be mainly attributed to the change of neutral IGM fraction. This evolution trend is in agreement with the Ly $\alpha$ fraction test which shows the fraction of LBGs with visible Ly $\alpha$ lines drops significantly at $z \gtrsim$ 7 (e.g., Schenker et al. 2014).

\subsection{Neutral IGM fraction at $z \sim 7$ with LAGER}

Following Ouchi et al. (2010) and Konno et al. (2014), we compare the Ly $\alpha$ luminosity densities at $z=6.9$ (in EoR) and at $z=5.7$ (when reionization is completed) to estimate the effective IGM transmission factor $T_{L y \alpha, z}^{\mathrm{IGM}}$. Assuming the stellar population, interstellar medium (ISM), and dust are similar at $z=6.9$ and $z=$ 5.7 , we obtain

$$
T_{z=6.9}^{\prime}=\frac{T_{L y \alpha, z=6.9}^{\mathrm{IGM}}}{T_{L y \alpha, z=5.7}^{\mathrm{IGM}}}=\frac{\rho_{z=6.9}^{L y \alpha} / \rho_{z=5.7}^{L y \alpha}}{\rho_{z=6.9}^{\mathrm{UV}} / \rho_{z=5.7}^{\mathrm{UV}}} .
$$

Here $\rho^{\mathrm{UV}}$ and $\rho^{\mathrm{Ly} \alpha}$ are UV and Ly $\alpha$ luminosity densities, respectively. The ratio of UV luminosity densities $\rho_{z=6.9}^{\mathrm{UV}} / \rho_{z=5.7}^{\mathrm{UV}}=0.63 \pm 0.09$ is obtained from Finkelstein et al. (2015). We obtain the ratio of observed Ly $\alpha$ luminosity densities (Col.-7 in Table 2) $\rho_{z=6.9}^{L y \alpha} / \rho_{z=5.7}^{L y \alpha}=0.24 \pm 0.03$. Thus we estimate $T_{z=6.9}^{\prime}=$ $0.38 \pm 0.07^{5}$ (c.f., $T_{z=7.3}^{\prime}=0.29$ from Konno et al. 2014 and $T_{z=6.6}^{\prime}=0.70 \pm 0.15$ from Konno et al. 2017).

Converting the Ly $\alpha$ emission line transmission factor to neutral IGM fraction $x_{H I}$ is model dependent (e.g., Santos 2004; McQuinn et al. 2007; Dijkstra et al. 2007). An important factor is the shift of the Ly $\alpha$ line with respect to the systemic velocity, which is widely observed and may be explained by $\operatorname{Ly} \alpha$ radiative transfer in a galactic wind or outflow. The Ly $\alpha$ velocity shift measurements of $z \sim 6-7$ galaxies are very limited and are mostly around 100-200 $\mathrm{km} \mathrm{s}^{-1}$ (Stark et al. 2015, 2017; Pentericci et al. 2016, but see two UV luminous galaxies with 400-500 $\mathrm{km} \mathrm{s}^{-1}$ reported by Willott et al. 2015). With the analytic model of Santos (2004), assuming a Ly $\alpha$ velocity shift of 0 and $360 \mathrm{~km} \mathrm{~s}^{-1}$, the value of $T_{z=6.9}^{\prime}$ $=0.38$ corresponds to $x_{H I} \sim 0.0$ and 0.6 , respectively.

By comparing our observed Ly $\alpha$ LF at $z=6.9$ to that predicted with the radiative transfer simulations by McQuinn et al. (2007, their Fig. 4), we obtain $x_{H I}$ $\sim 0.40-0.60$ at $z=6.9$. Similar results are obtained in the analytic studies considering ionization bubbles (Furlanetto et al. 2006; Dijkstra et al. 2007). We should note that these studies predicted a suppression of the luminosity function that is rather uniform across a wide range of luminosities (e.g., Sec. 4 of McQuinn et al. 2007). This suggests that the true distribution of ionized region sizes may differ appreciably from those used in literature (Furlanetto et al. 2006; Dijkstra et al. 2007; McQuinn et al. 2007). We conclude that the neutral hydrogen fraction is $x_{\mathrm{HI}} \sim 0.4-0.6$ at $z=6.9$, where both the uncertainties in the IGM transmission factor calculation and the theoretical model predictions are considered. The LBG Ly $\alpha$ fraction test by Schenker et al. (2014) yields a similar neutral hydrogen fraction $\left(x_{\mathrm{HI}}=\right.$ $\left.0.39_{-0.09}^{+0.08}\right)$ at $z \sim 7$. Note smaller $x_{\mathrm{HI}}=0.3 \pm 0.2$ at $z=$ 6.6 is obtained by Konno et al. (2017) with Subaru HSC and Suprime-Cam surveys.

\subsection{Ionized Bubbles at $z \sim r$}

The bump at the bright end of the Ly $\alpha$ LF is an indicator of large enough ( $>1 \mathrm{pMpc}$ radius) ionized bubbles, where the Hubble flow can bring Ly $\alpha$ photons out of resonance, thus leading to different evolution of Ly $\alpha$ LF at the bright end and the faint end. The uneven distribution of these LAEs may indicate ionized bubbles in a patchy reionization at $z \sim 7$. Larger LAGER samples in future will more definitely establish whether the degree of clustering in Figure 1 requires patchy reionization.

The origin of the ionized bubbles could be extrinsic or intrinsic, or both. From Malhotra \& Rhoads (2006), which gave the proper radius of a Strömgren sphere and the Gunn-Peterson effect optical depth of a LAE with outflow velocity $\Delta V$ in EoR, we know that even the brightest LAE can not produce a large enough ionized bubble to effectively reduce the optical depth to $\tau \sim 1$. We thus would expect extrinsic contribution from ad-

${ }^{5}$ Noe the error of $T_{z=6.9}^{\prime}$ is likely underestimated since we ignore the uncertainties from the faint-end slope of the Ly $\alpha$ LFs. 
Table 2

Best-fit Schechter Parameters and Ly $\alpha$ Luminosity Densities at $z \sim 5.7,6.6,6.9$ and 7.3.

\begin{tabular}{|c|c|c|c|c|c|c|c|c|}
\hline $\begin{array}{c}z \\
(1)\end{array}$ & $\begin{array}{l}\text { Area } \\
(2)\end{array}$ & $\begin{array}{l}\text { Volume } \\
\text { (3) }\end{array}$ & $\begin{array}{l}L \text { Fitted Range } \\
\text { (4) }\end{array}$ & $\begin{array}{c}\log _{10}\left[L_{L y \alpha}^{*}\right] \\
(5)\end{array}$ & $\begin{array}{c}\log _{10}\left[\Phi^{*}\right] \\
(6)\end{array}$ & $\begin{array}{c}\log _{10}\left[\rho_{L y \alpha}\right]^{\dagger} \\
(7)\end{array}$ & $\begin{array}{c}\log _{10}\left[\rho_{\text {Ly }}^{\text {Bright }}\right]^{\ddagger} \\
(8)\end{array}$ & $\begin{array}{l}\text { Reference } \\
\text { (9) }\end{array}$ \\
\hline & $\mathrm{deg}^{2}$ & {$\left[\mathrm{cMpc}^{3}\right]$} & & {$\left[\mathrm{erg} \mathrm{s}^{-1}\right]$} & {$\left[\mathrm{Mpc}^{-3}\right]$} & {$\left[\mathrm{erg} \mathrm{s}^{-1} \mathrm{Mpc}^{-3}\right]$} & {$\left[\mathrm{erg} \mathrm{s}^{-1} \mathrm{Mpc}^{-3}\right]$} & \\
\hline & & & & \multicolumn{5}{|c|}{ Two free parameters $\left[L_{L y \alpha}^{*}, \Phi^{*}\right]$ with fixed $\alpha=-2.5$} \\
\hline 5.7 & 13.8 & $1.16 \times 10^{7}$ & $42.4-44.0$ & $43.24 \pm 0.05$ & $-4.09_{-0.11}^{+0.13}$ & $39.54 \pm 0.01$ & 38.24 & Ouchi et al. (2008) + Konno et al. (2017) \\
\hline 6.6 & 21.2 & $1.91 \times 10^{7}$ & $42.4-44.0$ & $43.25_{-0.05}^{+0.06}$ & $-4.32 \pm 0.13$ & $39.33 \pm 0.02$ & 38.05 & Ouchi et al. (2010) + Konno et al. (2017) \\
\hline 6.9 & 2.0 & $1.26 \times 10^{6}$ & $42.65-43.25$ & $42.77_{-0.16}^{+0.32}$ & $-3.77_{-0.76}^{+0.54}$ & $* 38.92_{-0.04}^{+0.03}$ & *37.90 & This study \\
\hline & & & $42.65-43.65$ & $43.74_{-0.38}^{+0.55}$ & $-5.76_{-0.81}^{+0.72}$ & $38.77 \pm 0.05$ & 37.82 & This study \\
\hline 7.3 & 0.45 & $2.5 \times 10^{5}$ & 42.4-43.0 & $42.77_{-0.34}^{+1.23}$ & $\begin{array}{l}-4.09_{-1.91}^{+1.09} \\
\end{array}$ & $38.55_{-0.07}^{+0.08}$ & $* *$ & Konno et al. (2014) \\
\hline
\end{tabular}

The best-fit parameters in Col. 5 and Col. 6 are derived by fitting the Schechter function with fixed $\alpha=-2.5$ on the Ly $\alpha$ LFs listed in Col. 9 with the $\chi^{2}$-statistics. The errors in Col. 5-7 are estimated from their $1-\sigma$ confidence contours (e.g., Coe 2009) shown in the top panel of Fig. 3.

${ }^{\dagger}$ : Ly $\alpha$ luminosity densities integrated over the best-fit Schechter functions at $42.4<\log _{10} L_{L y \alpha}<44.0$ (Col.-7). Note the errors of Ly $\alpha$ luminosity densities are likely underestimated because we do not consider the uncertainties of the faint-end slope in that calculation. ¥: Ly $\alpha$ luminosity densities integrated at the bright-end $\left(43.3<\log _{10} L_{L y \alpha}<43.7\right.$, Col.-8).

*: The Ly $\alpha$ luminosity densities in this row is calculated by integrating the truncated Schechter function plotted as the red solid curve in Figure 2 and discussed in Section 3.2. We use the Ly $\alpha$ density in Col.-7 of this row to estimate the neutral IGM fraction at $z \sim 7$ in Section 4.2 .

**: The survey for $z=7.3 \mathrm{LAEs}$ does not have large enough volume to cover the bright end.

ditional satellite galaxies associated with the luminous LAEs. From Figure 1, we do see some such fainter nearby sources (e.g., the projected separations between LAE-1, 3 and 11 are $\lesssim 3.4$ arcmin, which corresponds to $1 \mathrm{pMpc}$ at $z=6.93)$. Further deeper NB imaging would enable us to probe the bubbles by detecting more fainter galaxies associated with the luminous LAEs.

The intrinsic explanation is that these luminous LAEs may be physically unusual objects, perhaps of a type not commonly found at lower redshift. For example, they could represent an AGN population that dominates over the ordinary star forming galaxies at $\lg _{10} L_{L y \alpha}>43.3$ (e.g., Matsuoka et al. 2016). Alternatively, they could be star forming galaxies where metallicities and/or dust abundances are low enough to enable considerably larger Ly $\alpha$ production or escape than is commonly seen at $z<$ 6 (e.g., Stark et al. 2015, 2017; Mainali et al. 2017). At $z \sim 7$, Bowler et al. (2014) find an excess of luminous LBGs where there should be an exponential cutoff at the bright-end of their UV LFs. NIR and FIR spectroscopic follow-up of these luminous LBGs show very large Ly $\alpha$ velocity offsets $\left(>300 \mathrm{~km} \mathrm{~s}^{-1}\right.$, Willott et al. 2015; Stark et al. 2017), which are significantly larger than the predicted velocity offsets for galaxies in EoR by Choudhury et al. (2015). Besides that, unusually strong carbon lines reported in two other LBGs at $z \sim 7$ (CIV $\lambda 1548 \AA$ in A1703-zd6 at $z=7.045$ and C III] $1909 \AA$ in EGS-zs8-1 at $z=7.73$ ) indicate unusual harder and more intense UV radiation than that of $z \sim 3$ LBGs (Stark et al. 2015, 2017). In addition, a strong He II line has been reported in the brightest Ly $\alpha$ emitter at $z=$ 6.6 (CR7), suggesting it could be powered by either Pop. III stars (Sobral et al. 2015) or perhaps accretion on to a direct collapse black hole (Pallottini et al. 2015).

Future deeper NB964 imaging and IR (NIR and/or FIR) spectroscopic observation will help us to determine the nature of these luminous LAEs, and probe the ionized bubbles in a patchy reionization at $z \sim 7$.

\section{CONCLUSION}

In this letter, we report the first results of our LAGER project, the discovery of 23 (22 new) candidate LAEs at $z \sim 7$. This is the largest sample to date of candidate
LAEs at $z \gtrsim 7$. Further more, thanks to the large survey volume of LAGER, we find 4 most luminous candidate LAEs at $z \sim 7$ with $\mathrm{L}(\operatorname{Ly} \alpha)=19-43 \times 10^{42} \mathrm{erg} \mathrm{s}^{-1}$. Compared to previous Ly $\alpha$ LFs at $z \gtrsim 6$, the Ly $\alpha$ LF of LAGER LAEs at $z \sim 7$ shows different evolution at the faint-end and at the bright-end, which indicates a large neutral hydrogen fraction $x_{\mathrm{HI}} \sim 0.4-0.6$ and the existence of ionized bubbles at $z \sim 7$. Our findings support the patchy reionization scenario at $z \sim 7$.

We thank the anonymous referee for careful and helpful comments which improve the manuscript. We acknowledge financial support from National Science Foundation of China (grants No. $11233002 \& 11421303$ ) and Chinese Top-notch Young Talents Program for covering the cost of the NB964 narrowband filter. J.X.W. thanks support from National Basic Research Program of China (973 program, grant No. 2015CB857005), and CAS Frontier Science Key Research Program QYCDJ-SSW-SLH006. Z.Y.Z acknowledges supports by the China-Chile Joint Research Fund (CCJRF No. 1503) and the CAS Pioneer Hundred Talents Program (C). L.I. is in part supported by CONICYT-Chile grants Basal- CATA PFB-06/2007, 3140542 and Conicyt-PIA-ACT 1417. C.J. acknowledges support by Shanghai Municipal Natural Science Foundation (15ZR1446600)

We thank Materion company for the manufacture of the NB964 filter, which made the LAGER project possible. We greatly appreciate the kind support from staffs at NOAO/CTIO to make our observations successful.

Based on observations at Cerro Tololo Inter-American Observatory, National Optical Astronomy Observatory (NOAO PID: 016A-0386, PI: Malhotra, and CNTAC PIDs: 2015B-0603 and 2016A-0610, PI: Infante), which is operated by the Association of Universities for Research in Astronomy (AURA) under a cooperative agreement with the National Science Foundation. Based in part on data collected at the Subaru Telescope and obtained from the Subaru-Mitaka-Okayama-Kiso Archive System (SMOKA), which is operated by the Astronomy Data Center, National Astronomical Observatory of Japan.

This project used data obtained with the Dark En- 
ergy Camera (DECam), which was constructed by the Dark Energy Survey (DES) collaboration. Funding for the DES Projects has been provided by the DOE and NSF (USA), MISE (Spain), STFC (UK), HEFCE (UK). NCSA (UIUC), KICP (U. Chicago), CCAPP (Ohio State), MIFPA (Texas A\&M), CNPQ, FAPERJ, FINEP (Brazil), MINECO (Spain), DFG (Germany) and the collaborating institutions in the Dark Energy Survey, which are Argonne Lab, UC Santa Cruz, University of Cambridge, CIEMAT-Madrid, University of Chicago, University College London, DES-Brazil Consortium, University of Edinburgh, ETH Zurich, Fermilab, University of Illinois, ICE (IEEC-CSIC), IFAE Barcelona, Lawrence Berkeley Lab, LMU Munchen and the associated Excellence Cluster Universe, University of Michigan, NOAO, University of Nottingham, Ohio State University, University of Pennsylvania, University of Portsmouth, SLAC National Lab, Stanford University, University of Sussex, and Texas A\&M University.

Facilities: Blanco (DECam), Subaru (Suprime-Cam)

\section{REFERENCES}

Annis, J., Soares-Santos, M., Strauss, M. A., et al. 2014, ApJ, 794,120

Baba, H., et al. 2002, ADASS XI, eds. D. A. Bohlender, D.

Durand, \& T.H. Handley, ASP Conference Series, Vol. 281, 298

Bagley, M.B., Scarlata, C., Henry, A., et al. 2017, ApJ, 837, 11

Bertin, E. 2010, Astrophysics Source Code Library, ascl:1010.068

Bolton, J.S., Haehnelt, M.G., Warren, S.J., et al. 2011, MNRAS, 416, L70

Bouwens, R.J., Illingworth, G.D., Oesch, P.A., et al. 2015, ApJ, 803, 34

Bowler, R.A.A., Dunlop, J.S., McLure, R.J., et al. 2014, MNRAS, 440, 2810

Coe D., arXiv:0906.4123

Choudhury, T.R., Puchwein, E., Haehnelt, M.G., et al. 2015 MNRAS, 452, 261

Dijkstra, M., Wyithe, J.S.B., Haiman, Z. 2007, MNRAS, 379, 253

Faisst, A.L., Capak, P., Carollo, C. M., et al. 2014, ApJ, 788, 87

Fan, X., Strauss, M. A., Becker, R. H., et al. 2006, AJ, 132, 117

Finkelstein, S. L., Ryan, R. E., Jr., Papovich, C., et al. 2015, ApJ, 810, 71

Furlanetto, S. R., Zaldarriaga, M., \& Hernquist, L. 2006 MNRAS, 365, 1012

Gehrels, N. 1986, ApJ, 303, 336

Haiman, Z., \& Cen, R. 2005, ApJ, 623, 627

Hibon, P., Cuby, J.-G., Willis, J., et al. 2010, A\&A, 515, 97

Hibon, P., Malhotra, S., Rhoads, J., Willott, C. 2011, ApJ, 741, 101

Hibon, P., Kashikawa, N., Willott, C., et al. 2012, ApJ, 744, 89

Hu, E. M., Cowie, L. L., Barger, A. J., et al. 2010, ApJ, 725, 394

Hu, W. D., Wang, J. X., Zheng, Z.Y., et al. 2017, submitted to ApJ, arXiv:1706.03586

Iye, M., Ota, K., Kashikawa, N., et al. 2006, Nature, 443, 186
Jensen, H., Laursen, P., Mellema, G., et al. 2013, MNRAS, 428, 1366

Jiang, L.H., Egami, E., Mechtley, M., et al. 2013, ApJ, 772, 99

Kashikawa, N., Shimasaku, K., Matsuda, Y., et al. 2011, ApJ,

734,119

Konno, A., Ouchi, M., Ono, Y., et al. 2014, ApJ, 797, 16

Konno, A., Ouchi, M., Shibuya, T., et al. 2017, submitted to PASJ, arXiv:1705.01222

Krug, H.B., Veileux, S., Tilvi, V., et al. 2012, ApJ, 745, 122

Madau, P. 1995, ApJ, 441, 18

Mainali, R., Kollmeier, J.A., Stark, D.P., et al. 2017, ApJ, 836, L14

Malhotra, S., \& Rhoads, J. E. 2006, ApJ, 647, L95

Malhotra, S., \& Rhoads, J. E. 2004, ApJ, 617, L5

Matsuoka, Y., Onoue, M., Kashikawa, N., et al. 2016, ApJ, 828, 26

Matthee, J., Sobral, D., Santos, S., et al. 2015, MNRAS, 451, 400

McCracken, H. J., Milvang-Jensen, B., Dunlop, J., et al. 2012, A\&A, 544, 156

McQuinn, M., Hernquist, L., Zaldarriaga, M., \& Dutta, S. 2007, MNRAS, 381, 75

Muzzin, A., Marchesini, D., Stefanon, M., et al. 2013, ApJS, 206, 8

Ota, K., Iye, M., Kashikawa, N., et al. 2008, ApJ, 677, 12-26

Ota, K., Iye, M., Kashikawa, N., et al. 2010, ApJ, 722, 803

Ota, K., Iye, M., Kashikawa, N., et al. 2017, submitted to ApJ, arXiv: 1703.02501

Ouchi, M., Shimasaku, K., Akiyama, M., et al. 2008, ApJS, 176, 301-330

Ouchi, M., Shimasaku, K., Furusawa, H., et al. 2010, ApJ, 723, 869

Pallottini, A., Ferrara, A., Pacucci, F., et al. 2015, MNRAS, 453, 2465

Pentericci, L., Carniani, S., Castellano, M., et al. 2016, ApJ, 829, 11

Planck Collaboration, Ade, P. A. R., Aghanim, N., et al. 2016, A\&A, 594, A13

Rhoads, J. E., Hibon, P., Malhotra, S., et al. 2012, ApJ, 752, L28

Rhoads, J. E., \& Malhotra, S. 2001, ApJ, 563, L5

Santos, M. R. 2004, MNRAS, 349, 1137

Santos, S., Sobral, D., \& Matthee, J. 2016, MNRAS, 463, 1678

Schenker, M. A., Ellis, R. S., Konidaris, N. P., Stark, D. P. 2014 ApJ, 795, 20

Shibuya, T., Kashikawa, N., Ota, K., et al. 2012, ApJ, 752, 114

Sobral, D., Matthee, J., Darvish, B., et al. 2015, ApJ, 808, 139

Stark, D. P., Richard, J., Charlot, S., et al. 2015, MNRAS, 450, 1846

Stark, D. P., Ellis, R.S., Charlot, S., et al. 2017, MNRAS, 464, 469

Stern, D., Yost, S. A., Eckart, M. E., et al. 2005, ApJ, 619, 12

Suchyta, E., Huff, E. M., Aleksić, J., et al. 2016, MNRAS, 457, 786

Tilvi, V., Rhoads, J.E., Hibon, P., et al. 2010, ApJ, 721, 1853

Tilvi, V., Pirzkal, N., Malhotra, S., et al. 2016, ApJ, 827, 14

Valdes, F., Gruendl, R., DES Project 2014, ASPC, 485, 379

Willott, C. J., Carilli, C.L., Wagg, J., Wang, R. 2015, ApJ, 807, 180

Zheng, Z.Y., Malhotra, S., Rhoads, J. E., et al. 2016, ApJS, 226, 23 